\title{
Concurrent esterification and $\mathrm{N}$-acetylation of amino acids with orthoesters: A useful reaction with interesting mechanistic implications
}

\author{
Sarah Gibson, Dickie Romero, Hollie K. Jacobs, and Aravamudan S. Gopalan * \\ Department of Chemistry and Biochemistry, New Mexico State University Las Cruces, NM \\ 88003-8001
}

\begin{abstract}
The concurrent esterification and $N$-acetylation of amino acids has been studied with triethyl orthoacetate (TEOA) and triethyl orthoformate (TEOF). In a surprising finding, only one equivalent of TEOA in refluxing toluene was necessary to convert L-proline and L-phenylalanine to the corresponding $N$-acetyl ethyl esters in good yield. The same transformation using TEOF was not effective. Stereochemical outcome and stoichiometric studies as well as structural variation of the amino acids in this reaction provided unexpected mechanistic insight.
\end{abstract}

\section{Keywords}

orthoester; amino acid; esterification; acetylation; mechanism

\begin{abstract}
Orthoesters have been widely used in organic synthesis for a variety of reactions.i However, the difference in reactivity between the most commonly used orthoesters, triethyl orthoacetate (TEOA) and triethyl orthoformate (TEOF), has not been clearly appreciated. A while ago, we reported that TEOA and trimethyl orthoacetate (TMOA) were more effective and superior in the esterification of carboxylic acids and sulfonic acids under mild reaction conditions than TEOF.ii Recently, esterification of carboxylic acids using TMOA or TEOA under solvent-free conditions in a microwave to yield the corresponding methyl or ethyl esters has been reported.iii The use of TEOA or TMOA for the esterification of carboxylic, phosphinic and phosphonic acids in ionic liquids $\left(80^{\circ} \mathrm{C}, 100 \mathrm{~min}\right)$ has also been reported.iv Orthoesters have been used in the esterification of $N$-acylamino acids. ${ }^{v}$ In the microwaveassisted esterification of various $N$-protected amino acids, TEOA was found to better effect the esterification than TEOF. ${ }^{\mathrm{vi}}$
\end{abstract}

The reaction of amines with orthoesters is quite complicated and is dependent on a number of variables including the nature of the amine and the orthoester, stoichiometry of the reagents, presence of an acid catalyst, and the reaction conditions, leading to the formation of a variety of nitrogen-containing products including amides, imidates, amidines and orthoamines. ${ }^{\text {vii }}$ Formylation of several amino acid ester hydrochlorides using TEOF (3 eq) at reflux $\left(145^{\circ} \mathrm{C}\right)$ for 1 hour in the absence of solvent without racemization has been

\footnotetext{
*Tel.: 1-575-646-2589; fax: 1-575-646-2649; agopalan@nmsu.edu.

Publisher's Disclaimer: This is a PDF file of an unedited manuscript that has been accepted for publication. As a service to our customers we are providing this early version of the manuscript. The manuscript will undergo copyediting, typesetting, and review of the resulting proof before it is published in its final citable form. Please note that during the production process errors may be discovered which could affect the content, and all legal disclaimers that apply to the journal pertain.
} 
reported. ${ }^{\text {viii }}$ There has also been a brief report on the acetylation of DL-leucine ethyl ester with TEOA to give the $N$-acetyl derivative. ${ }^{\text {ix }}$

There is very little known on the reactivity of TEOF and TEOA with free amino acids. In one isolated report, it was disclosed that proline upon reflux in dimethyl acetamide with TEOA ( $1.5 \mathrm{eq}$ ) for 15 minutes gave $N$-acetyl proline ethyl ester in $80-85 \%$ yield. ${ }^{\mathrm{x}}$ Unfortunately, this study was limited to one example, which prevents understanding the usefulness and limitations of this type of transformation. In this communication, we would like to report the results of our study on the reaction of amino acids using TEOA and TEOF. This study not only discloses some interesting mechanistic implications of this reaction but also clearly delineates the synthetic utility and differences in reactivity between TEOA and TEOF.

We began our investigation by trying to study and understand the differential reactivity, if any, of TEOA and TEOF on the acetylation/formylation of amino acid esters (Scheme 1). LProline methyl ester hydrochloride was reacted with one equivalent of TEOA or TEOF in toluene at room temperature for 24 hours. In the case of TEOF, the reaction did not proceed but led to the isolation of unreacted starting material. None of the $N$-formyl product was isolated. To our surprise, the corresponding reaction with TEOA was successful even under such moderate reaction conditions. The desired $N$-acetyl derivative was isolated in $73 \%$ yield. When the same reactions were carried out using refluxing toluene for 24 hours, both formylation and acetylation could be accomplished in good yields. It is important to mention that our attempts to acetylate underivatized proline at room temperature using TEOA were not successful. This lack of reactivity may be due to the absence of a strong acid catalyst in the reaction media.

In the case of L-phenyalanine ethyl ester hydrochloride, acetylation with TEOA was observed at room temperature; however, the conversion was low and the desired product was isolated along with unreacted starting material (Scheme 1). In constrast, the use of TEOF under identical conditions gave no desired formylation. The same reactions when carried out in refluxing toluene gave the desired products in good yields, with the acetylation being quantitative under these conditions. As in the case of L-proline, no acetylation was observed when unprotected L-phenylalanine was treated with TEOA at room temperature. Our studies clearly establish that acetylation of amino acid esters with TEOA occurs more readily than the corresponding formylation with TEOF. Further, it is important to note that the selective acetylation or formylation of the unprotected amino acids could not be accomplished with either of these reagents.

Given the greater reactivity of the amino group over the carboxylic acid, one would predict that when amino acids are treated with TEOA, initial reaction would occur at the amine site. The question was whether amino acids such as proline could be converted to the corresponding $\mathrm{N}$-acetyl ester derivatives stepwise, via the intermediacy of the $\mathrm{N}$-acetyl intermediate. Our study of this transformation resulted in some surprising findings.

When L-proline was heated with TEOA (1 eq.) in refluxing toluene for 24 hours, to our surprise the major product that was isolated in good yields was the $N$-acetyl ethyl ester (Table 1). Aqueous work-up followed by distillation under reduced pressure, gave $\mathrm{N}$-acetyl proline ethyl ester as the only product in $79 \%$ yield. Later, it was found that the product could be obtained in high yield and purity by removal of the reaction solvent in vacuo followed by filtration of the crude product through a short silica gel column using chloroform. ${ }^{\mathrm{xi}}$ The corresponding reaction with TEOF even when carried out with 5 equivalents of the reagent gave a complex mixture of products which were not easily separated. The same reaction was then carried out with L-phenylalanine. Once again, using 
TEOA (1 eq.) in refluxing toluene, the $N$-acetyl ethyl ester of phenylalanine was isolated in $87 \%$ yield. It is noteworthy that the TEOA reaction with L-proline gave $N$-acetyl ethyl ester of proline without racemization $\left([\alpha]_{\mathrm{D}}-95^{\circ}(c 1.0, \mathrm{EtOH})\right.$, lit. $^{10}[\alpha]_{\mathrm{D}}-90^{\circ}(c 1, \mathrm{EtOH})$. However, the product obtained from the reaction of L-phenylalanine with TEOA exhibited no optical rotation, indicating it was racemic!

The observed stoichiometry and stereochemical outcome of the TEOA reaction with proline and phenylalanine was surprising. We had anticipated that an excess of TEOA would be needed for the simultaneous acetylation and esterification reaction. In order to gain some mechanistic insight into the reaction of TEOA with amino acids, a study of the stoichiometry of these reactions was conducted (Figure 1). The reactions of proline and phenylalanine with varying equivalents of TEOA were conducted in refluxing toluene for 24 hours. In the case of proline, good yields of product could be obtained with one equivalent of TEOA. However, the yield was slightly higher when the reaction was conducted with two equivalents of TEOA or more. A different trend was observed for the reaction of phenylalanine with TEOA. Optimal yields were observed with approximately one equivalent of TEOA. A decrease in yield was observed when an excess of TEOA was used indicating that the product was undergoing further reaction. These studies clearly show that only one equivalent of TEOA is required for both esterification and acetylation of proline or phenylalanine.

To rationalize the results of our studies of the reactions of TEOA with L-proline and Lphenylalanine, we propose the following mechanism (Scheme 2). TEOA first reacts with the more reactive amino group to give an imidate ester, which undergoes cyclization to give the oxazolidinone I. Subsequent ring opening of I with ethanol formed in the first step results in formation of the $N$-acetyl ester product. In the case of primary amino acids like phenylalanine, the intermediate oxazolidinone undergoes rapid racemization through tautomerization to the enol form. Racemization of amino acids through their oxazolidinone is known. ${ }^{\text {xii }}$ For proline, the reaction with TEOA proceeds with no loss of enantiomeric purity. Presumably, the bicyclic oxazolidinone II derived from proline undergoes ring opening with ethanol much faster than any racemization process, which could be disfavored due to energy constraints offered by the bicyclic ring of the intermediate. In any case, only one equivalent of TEOA is necessary in this reaction to achieve both $\mathrm{N}$-acetylation and esterification.

In general, $\alpha$-amino acids (Table 1, entries 1-6) react with one equivalent of TEOA to give good yields of the corresponding $N$-acetyl esters. The corresponding methyl esters can be obtained using TMOA. ${ }^{\text {xiii }}$

If our proposed mechanism were valid, one would predict that the reaction of $\beta$-amino acids and other amino acids with TEOA could follow a different pathway that would involve independent acetylation and esterification events. If the formation of oxazolidinone $\mathbf{I}$ is not feasible, the reaction should require a minimum of two equivalents of TEOA for completion under comparable reaction conditions. In order to test our hypothesis, the reaction of TEOA with racemic 2-, 3- and 4-piperidinecarboxylic acid was examined (Table 1). Only in the case of piperidine 2-carboxylic acid formation of the 5-membered oxazolidinone intermediate $\mathbf{I}$ is feasible. The reaction of piperidine 2-carboxylic acid with TEOA with one equivalent of TEOA gave $85 \%$ of the desired $N$-acetyl ethyl ester. In contrast, 3 - and 4piperidine carboxylic acids gave very little of the desired products upon treatment with one equivalent TEOA under similar reaction conditions. In both cases, good yields of the desired products could be obtained using 5 equivalents of TEOA in refluxing toluene or neat TEOA at high temperatures. These conditions are more similar to those required for esterification of simple carboxylic acids with TEOA. ${ }^{2}$ It is interesting to point out that in the case of 3- 
aminobenzoic acid, the product isolated on reaction with TEOA ( 2 eq.) was the imidate ethyl ester, and not the $N$-acetyl ester. This supports our mechanistic hypothesis that an imidate ester is involved in this process.

In conclusion, we have shown that TEOA is an effective reagent for the concurrent and one pot $\mathrm{N}$-acetylation and esterification of amino acids under neutral conditions. TEOF exhibits lower reactivity and performs poorly in this type of transformation. In the case of $\alpha$-amino acids, one equivalent of TEOA in refluxing toluene is sufficient to give the corresponding $\mathrm{N}$ acetyl ethyl esters in good yields. Stoichiometric studies indicate that the mechanistic pathway in the reaction of $\alpha$-amino acids involves the intermediacy of an oxazolidinone. This is not so where geometric constraints disallow the formation of the oxazolidinone intermediate. In these cases, the desired $N$-acetyl esters can be prepared in good yields by using a larger excess of TEOA. It is important in emphasize that the choice of TEOA or TEOF is important and may govern the success of the desired transformation. It is clear that further studies are required to delineate the differences in reactivity between TEOA and TEOF with other substrates and transformations. Also, the use of other orthoesters may allow variation of the acyl or ester groups that are incorporated.

\section{Acknowledgments}

This research was supported by grants from the National Institutes of Health 1SC3GM084809-01 and GMO7667-34 (fellowship to SG and DR). The NSF GRFP is also thanked for a fellowship to SG.

\section{References}

i. a) Taylor, RT. Triethyl orthoformate. In: Paquette, LA., editor. Encyclopedia of reagents for organic synthesis. Vol. 7. John Wiley \& Sons; New York: 1995. p. 5102-5105.b) Ziegler, FE.; Belema, M.; Harran, PG.; Kover, RX. Triethyl orthoacetate. In: Paquette, LA., editor. Encyclopedia of reagents for organic synthesis. Vol. 7. John Wiley \& Sons; New York: 1995. p. 5099-5102. c) Pavlova LA, Davidovich YA, Rogozhin SV. Russian Chem Rev 1986;55(11):1026-1041.

ii. Trujillo J, Gopalan AS. Tetrahedron Lett 1993;34:7355-7358.

iii. Lee JC, Song I, Park JY. Synth Commun 2002;32:2209-2213.

iv. Yoshino T, Imori S, Togo H. Tetrahedron 2006;62:1309-1317.

v. Rogozhin SV, Davidovich YA, Korshak VV. Izv Akad Nauk SSSR, Ser Khim 1969;12:2858.

vi. Zhang S, Arvidsson PI. Int J Pept Res Ther 2008;14:219-222.

vii. a) Swaringen RA Jr, Eaddy JF, Henderson TR. J Org Chem 1980;45:3986. b) Taylor EC, Ehrhart WA. J Org Chem 1963;28:1108. c) Roberts RM, Vogt PJ. J Am Chem Soc 1956;78:4778.

viii. Chancellor T, Morton C. Synthesis 1994:1023.

ix. Rogozhin SV, Davidovich YA, Korshak VV. Izv Akad Nauk SSSR, Ser Khim 1970;3:727.

x. Korshak VV, Rogozhin SV, Davidovich YA. Izv Akad Nauk SSSR, Ser Khim 1969:977.

xi. Synthesis of $N$-acetyl proline ethyl ester: A mixture of L-proline $(0.2 \mathrm{~g}, 1.7 \mathrm{mmol})$ and triethyl orthoacetate $(0.6 \mathrm{~mL}, 3.5 \mathrm{mmol})$ in toluene $(1 \mathrm{~mL})$ was refluxed under nitrogen for 24 hours. After cooling to room temperature, the toluene was removed under reduced pressure. The residue was dissolved in $\mathrm{CHCl}_{3}$ and filtered through a PrepSep solid phase extraction tube (Fisher Scientific, silica gel, $1.0 \mathrm{~g})$. The solvent was removed in vacuo to afford the product $(0.265 \mathrm{~g}$, $84 \%)$.

xii. Wang XJ, Yang Q, Liu F, You QD. Synth Commun 2008;38:1028.

xiii. Spectroscopic analyses of all compounds are in agreement with the assigned structures 


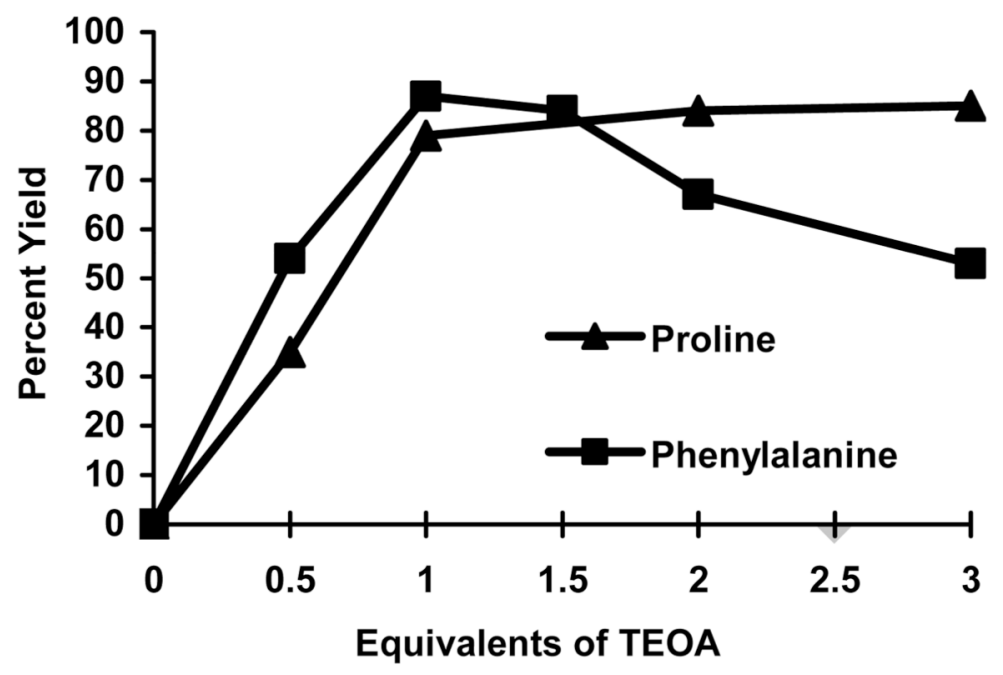

Figure 1. Reaction stoichiometry of phenylalanine and proline with TEOA 


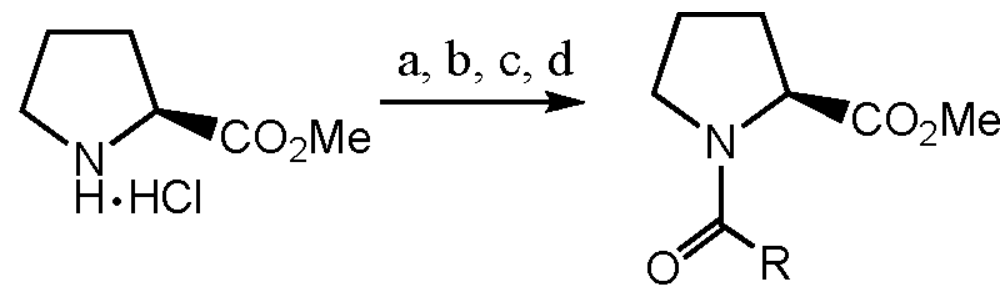

a) 1 eq. TEOA, toluene, rt, $24 \mathrm{~h}, 73 \%$

b) 1 eq. TEOA, toluene, $110^{\circ} \mathrm{C}, 24 \mathrm{~h} .89 \%$

c) 1 eq. TEOF, toluene, $\mathrm{rt}, 24 \mathrm{~h}$, no reaction

d) 1 eq. TEOF, toluene, $110^{\circ} \mathrm{C}, 24 \mathrm{~h}, 81 \%$

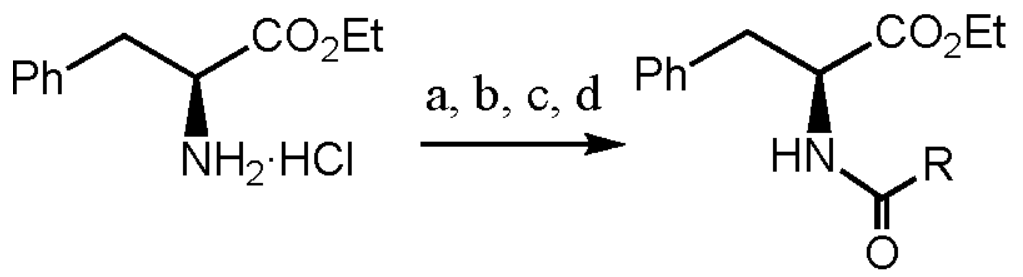
a) $26 \%$
b) $100 \%$
c) no reaction
d) $69 \%$

Scheme 1. Reaction of amino acid esters with TEOA and TEOF 
<smiles>[R]OC(C)(OCC)OCC</smiles><smiles>[R][C@]([2H])(N=C(C)OCC)C(=O)O</smiles><smiles>C=C</smiles>

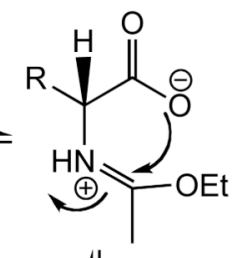<smiles>[R]C12C(=O)C(CCO)(CC1CCO)OC2(C)C</smiles><smiles>[R]c1nc(C)oc1O</smiles><smiles>[R][C@]1([2H])N=C(C)OC1=O</smiles><smiles>[R][C@H]1N[C@](C)(OCC)OC1=O</smiles><smiles>[R][C@@H](NC(C)=O)C(=O)OCC</smiles>

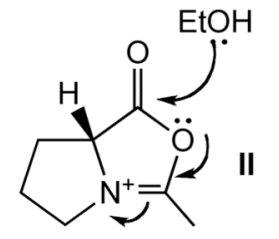

Scheme 2.

Proposed mechanism for the reaction of TEOA with amino acids. 


\section{Table 1}

Reactions of amino acids with TEOA

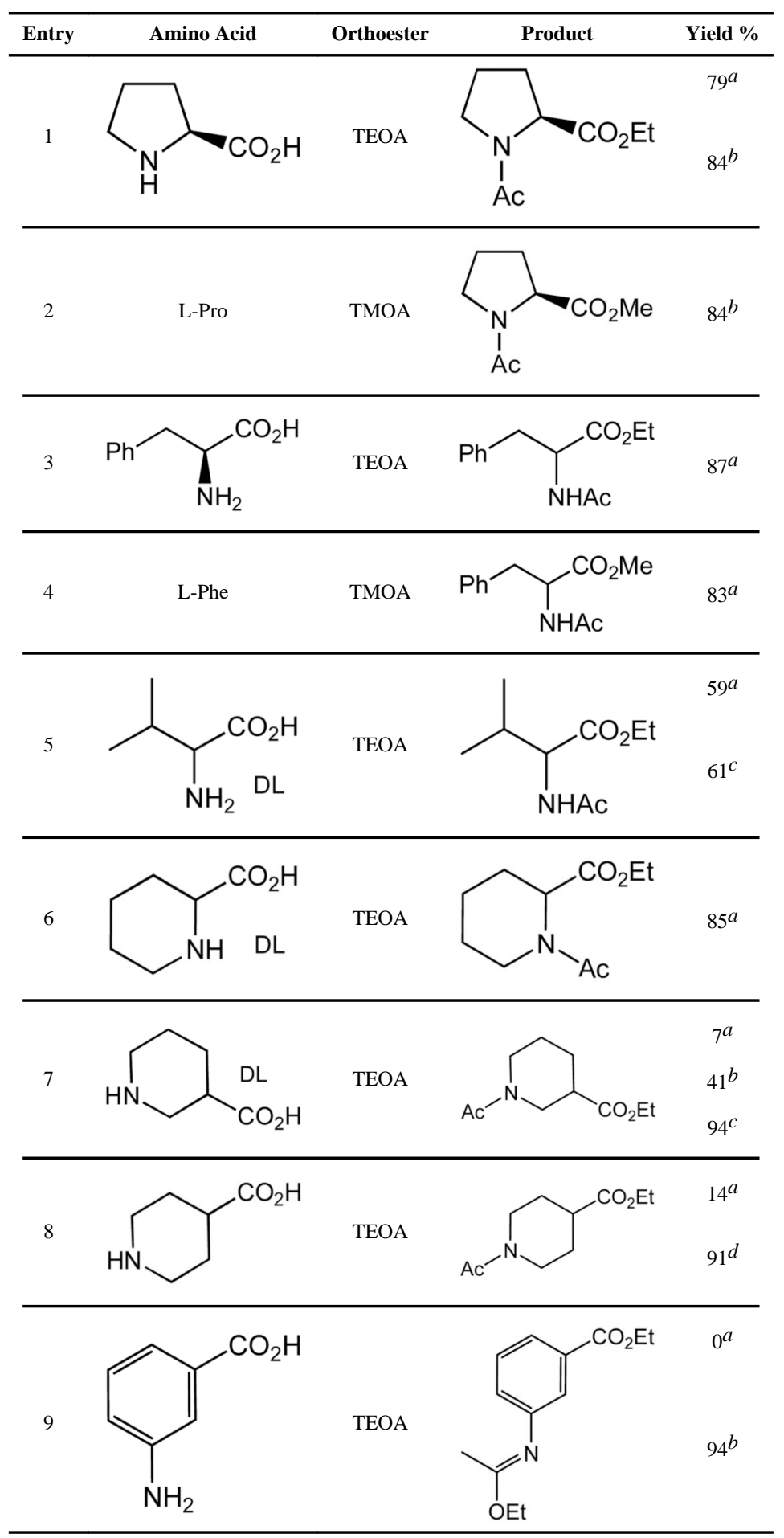

$a_{1-1.2 \text { eq. TEOA, toluene, } 110^{\circ} \mathrm{C}, 24 \mathrm{~h}}$ 
$b_{2}$ eq. TEOA, toluene, $110^{\circ} \mathrm{C}, 24 \mathrm{~h}$

${ }^{c} 5$ eq. TEOA, toluene, $110^{\circ} \mathrm{C}, 24 \mathrm{~h}$

$d_{5 \text { eq. TEOA, } 125^{\circ} \mathrm{C}, 24 \mathrm{~h}}$ 